\title{
CHEK2 variants in susceptibility to breast cancer and evidence of retention of the wild type allele in tumours
}

\author{
N Sodha*,, S Bullock², R Taylor³, G Mitchell',2, B Guertl-Lackner4, RD Williams', S Bevan², K Bishop', \\ S McGuire ${ }^{2}$, RS Houlston ${ }^{2}$ and RA Eeles ${ }^{1,2}$
}

'Royal Marsden NHS Trust, Downs Road, Sutton, Surrey SM2 5PT, UK; '2Section of Cancer Genetics, Institute of Cancer Research, Sutton, Surrey SM2 5NG, UK; ${ }^{3}$ Medical Genetics Unit, St George's Hospital, London, SWI 7 ORE, UK; ${ }^{4}$ Section of Molecular Carcinogenesis, Institute of Cancer Research, Sutton, Surrey SM2 5NG, UK; ${ }^{5}$ Section of Paediatric Oncology, Institute of Cancer Research, Sutton, Surrey SM2 5NG, UK

\begin{abstract}
We have recently shown that the CHEK2* I I00delC mutation acts as a low penetrance breast cancer susceptibility allele. To investigate if other CHEK2 variants confer an increased risk of breast cancer, we have screened an affected individual with breast cancer from 68 breast cancer families. Five of these individuals were found to harbour germline variants in CHEK2. Three carried the I I00delC variant (4\%). One of these three individuals also carried the missense variant, Arg $80 \mathrm{His}$. In the other two individuals, missense variants, Arg I I G Gly and Arg I37Gln, were identified. These two missense variants reside within the Forkhead-associated domain of CHEK2, which is important for the function of the expressed protein. None of these missense variants were present in 300 healthy controls. Microdissected tumours with a germline mutation showed loss of the mutant allele suggesting a mechanism for tumorigenesis other than a loss of the wild type allele. This study provides further evidence that sequence variation in CHEK2 is associated with an increased risk of breast cancer, and implies that tumorigenesis in association with CHEK2 mutations does not involve loss of the wild type allele.
\end{abstract}

British Journal of Cancer (2002) 87, |445 - |448. doi:I0.1038/sj.bjc.6600637 www.bjcancer.com

(C) 2002 Cancer Research UK

Keywords: breast cancer; CHEK2; mutation

About $10 \%$ of breast cancer has a genetic predisposition, however, germline mutations in BRCA1, BRCA2, TP53, PTEN and LKB1 only account for between $20-25 \%$ of the familial clustering (Easton, 1999). A recent model of breast cancer susceptibility suggests a polygenic basis for the wide variation in familial risk whereby the individually small effects of many genes combine multiplicatively (Antoniou et al, 2001). We have recently found evidence for this model with the observation that the 1100delC variant in CHEK2 which is carried by $\sim 1 \%$ of the population confers a 1.7 -fold increased risk of breast cancer (Meijers-Heijboer et al, 2002).

CHEK2 functions downstream of ATM (ataxia telangiectasiamutated protein) in response to DNA damage (Chaturvedi et al, 1999) to phosphorylate TP53 (Chehab et al, 2000) and BRCA1 (Lee et al, 2000), therefore regulating the tumour suppressor functions of these proteins. The protein contains functionally important 60 amino acid FHA domain (residues 115-175) and a kinase domain (residues 226-486) (Matsuoka et al, 1998).

To investigate if variants of CHEK2 other than 1100delC confer an increased risk of breast cancer, we have screened a series of 68 familial breast cancer cases, which had been screened in the Regional Genetics Service and found to be negative for mutations in $B R C A 1$ and BRCA2.

\section{MATERIALS AND METHODS}

\section{Patients}

EDTA-venous blood samples were collected from 68 familial breast cancer cases attending Genetics Clinics within the Royal Marsden NHS Trust and the South Thames Regional Genetics Unit. All families had at least two individuals affected with breast cancer. The breast cancer was verified by histological reports. Samples were obtained with informed consent and local ethical approval in accordance with the tenets of the Helsinki Declaration. DNA was extracted from EDTA-blood samples using a standard sucrose lysis method.

\section{METHODS}

\section{Mutational analysis of BRCA1 and BRCA2}

Mutational analysis of $B R C A 1$ and $B R C A 2$ was undertaken using a combination of the protein truncation test (PTT) (Hogervorst et al, 1995), and conformation sensitive gel electrophoresis (CSGE) (Ganguly et al, 1993). Exons 10 and 11 of BRCA2 and exon 11 of BRCA1 were screened by PTT. Exon 9 of BRCA2 and exons 2 and 20 of BRCA1 were screened by CSGE.

\section{Mutational analysis of CHEK2}

The search for germline mutations in CHEK2 was performed using CSGE as described previously (Sodha et al, 2002). Samples that showed variant migration bands were sequenced by direct sequencing using the ABI BigDye cycle sequencing kit with dye-labelled 
terminators. These labelled products were then run on an ABI 310 sequencer (Applied Biosystems).

\section{Loss of heterozygosity ( $\mathrm{LOH})$ studies}

Allelic imbalance in tumours from individuals with germline mutations in CHEK2 was assessed using the microsatellite marker, D22S275, which maps to intron 4 of CHEK2. The relevant exons were also amplified by PCR from the tumour DNA and sequenced. DNA was obtained from paraffin-embedded tissue by dewaxing with xylene, digesting with $10 \mathrm{mM}$ Tris- $\mathrm{HCl}$ (pH 7.5), $1 \mathrm{mM}$

Table I Family details of the 68 patients studied

\begin{tabular}{lccc}
\hline No. of families & $\begin{array}{c}\text { No. of breast } \\
\text { cancer cases }\end{array}$ & $\begin{array}{c}\text { No. of these } \\
\text { families with } \\
\text { ovarian cancer }\end{array}$ & $\begin{array}{c}\text { Median age range } \\
\text { of proband } \\
\text { (years; range) }\end{array}$ \\
\hline 16 & $4+$ & 5 & $48(35-58)$ \\
20 & 4 & 5 & $49(33-56)$ \\
16 & 3 & 3 & $42(31-51)$ \\
16 & 2 & 3 & $44(32-59)$ \\
\hline
\end{tabular}

EDTA, $15 \%\left(\mathrm{w} \mathrm{v}^{-1}\right)$ SDS and $500 \mu \mathrm{g} \mathrm{ml}^{-1}$ proteinase $\mathrm{K}$ for $4 \mathrm{~h}$ at $56^{\circ} \mathrm{C}$, followed by phenol-chloroform extraction and sodium acetate, ethanol precipitation.

\section{Statistical analysis}

The $95 \%$ confidence interval $(95 \% \mathrm{CI})$ of the estimate of the frequency of CHEK2 mutations in breast cancer cases was estimated from the binomial distribution. All statistical manipulations were undertaken using the statistical software programme STATA (Version 6.0, Stata Corporation, College Station, Texas, TX 77840, USA; http://www.stata.com).

\section{RESULTS}

Table 1 shows the family details of the 68 breast cancer cases studied. All had a strong family history of breast cancer or breast-ovarian cancer. The median age at diagnosis of all cases was 47 years.

Seventeen of the 68 patients were fully screened for mutations in $B R C A 1$ and BRCA2. The others were screened for mutations in exons 9-11 of BRCA2 and exons 2, 11 and 20 of BRCA1. No pathogenic mutations were detected in any of the 68 patients. This

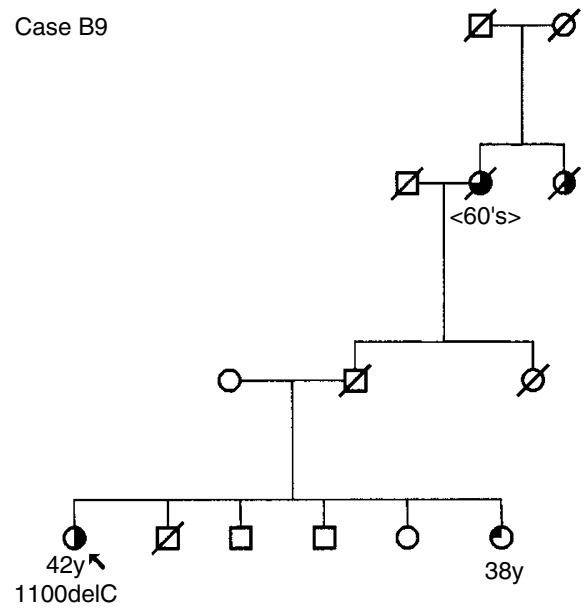

Case G11

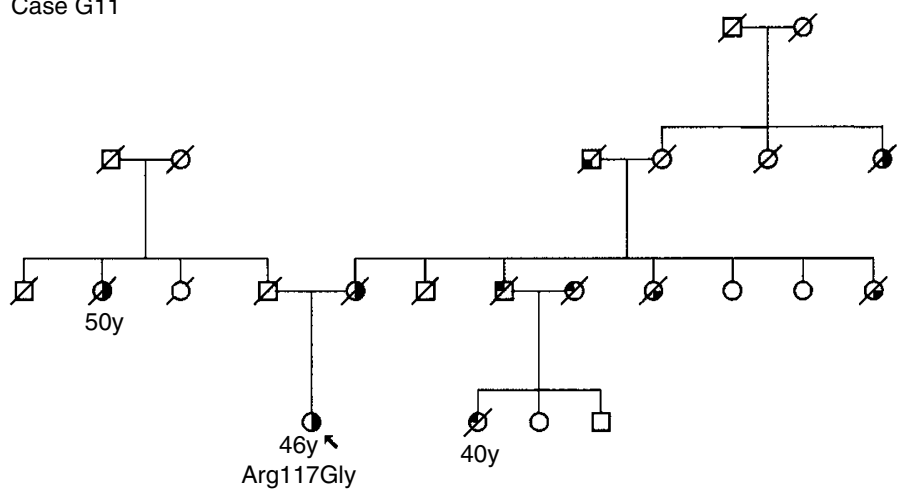

Case D11

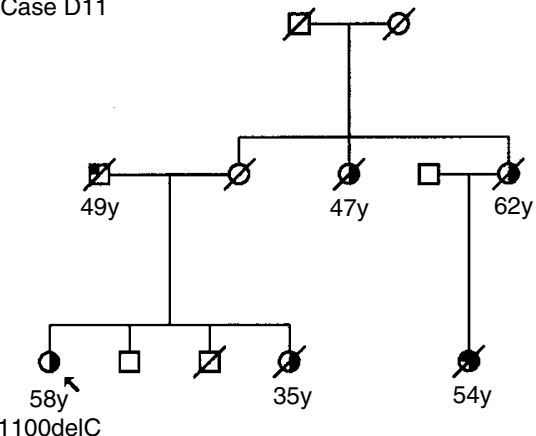

Case F4

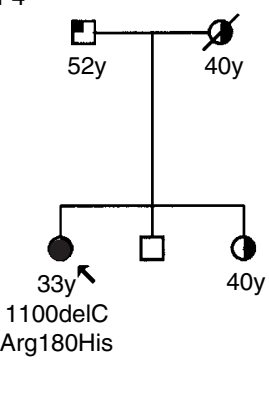

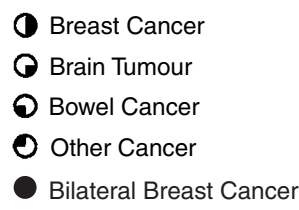

Figure I The pedigrees of the breast cancer cases with germline mutations in CHEK2 (individuals harbouring the mutation are arrowed). The trees have been altered to preserve anonymity, but not alter the meaning of the report. 
(A)

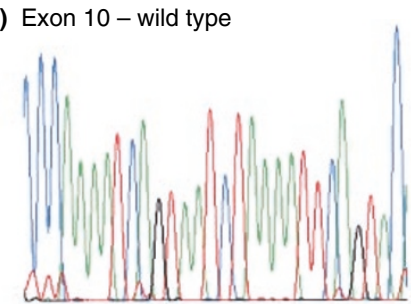

(B) Exon 10-D11 germline
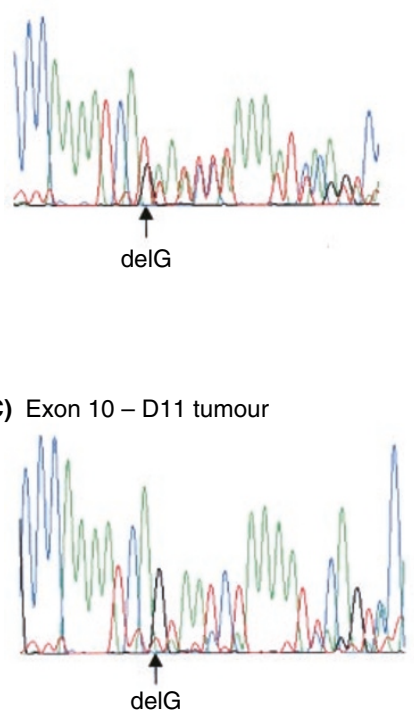

(C) Exon $10-$ D11 tumour
(D) Exon 2 - wild type

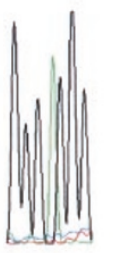

(E) Exon 2 - G11 germline

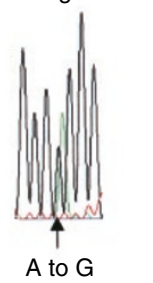

(F) Exon 2-G11 tumour

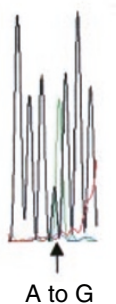

Figure 2 Partial sequences of exon 10 and exon 2 of CHEK2. (A) wild type reverse sequence of exon 10 (B) germline reverse sequence of exon 10 from case DII $(\mathbf{C})$ reverse sequence of exon 10 from tumour DNA from case DII (D) wild type forward sequence of exon 2 (E) germline forward sequence of exon 2 from case GI I (F) forward sequence of exon 2 from tumour DNA from case GII. The wild type allele is retained and there is a low level signal of the mutant allele in both the sequences of tumour DNA in (C) and (F) (arrowed).

analysis is estimated to detect approximately $75 \%$ of the reported BRCA1 mutations and $50 \%$ of the BRCA2 gene. (BIC database: www.nhgri.nih.gov/Intramural_research/Lab_transfer/Bic/).

The full coding sequence of CHEK2 was screened for mutations in all 68 patients. Six sequence variants were identified. Figure 1 shows the family histories of the individuals carrying the variants. Three of the cases, B9, F4 and D11, carried the 1100delC variant. One of these cases, F4, also carried the missense variant, Arg180His. The other two individuals, G11 and $\mathrm{H} 10$, carried sequence changes in exon $2-$ Arg117Gly and Arg137Gln.

To establish whether the CHEK2 missense variants identified represent polymorphisms, 300 healthy controls were screened for these sequence changes. None of the controls were found to harbour these changes. Unfortunately, germline DNA was not available from other members in any of the three families to examine whether either variant was carried by any other family member affected with breast cancer.

Paraffin embedded tissue was available from the two patients with the 1100delC variant (B9 and D11) and the patient with the Arg117Gly variant (G11). Analysis of tumour DNA from B9 showed no evidence of allelic imbalance by sequencing. Sequencing of relevant exons from tumour DNA from D11 and G11, however,
(A) F4 - germline DNA

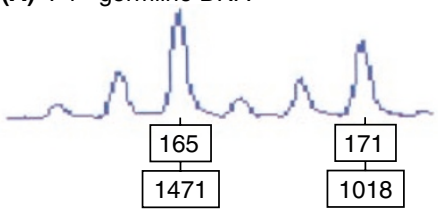

(E) G11 - germline DNA

(B) B9 - germline DNA

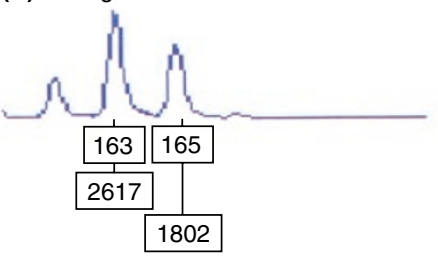

(F) G11 - tumour DNA

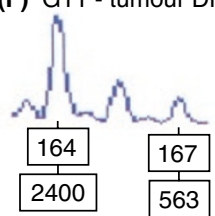

(C) D11 - germline DNA

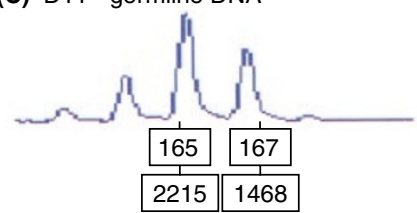

(D) D11 - tumour DNA

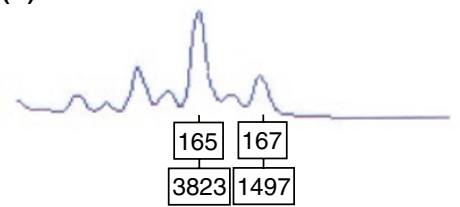

Figure 3 Genescans of the microsatellite marker D22S275. (A-C) Germline DNA of individuals with the II00delC variant (D) tumour DNA of case DII (E) germline DNA of case GII who harbours the variant Arg I I G Gly and (F) tumour DNA of GII. There is a loss of heterozygosity in tumour DNA from both the cases.

showed that the mutant allele was lost and the wild type allele retained (Figure 2).

Figure 3 shows D22S275 genotypes of patients F4, B9 and paired normal -tumour DNA samples from patients D11 and G11. We have previously shown that the $165 \mathrm{bp}$ allele of D22S275 is associated with the 1100delC variant (Meijers-Heijboer et al, 2002) and in this study all three patients who harbour this sequence change (B9, F4, D11) possessed this allele. Figure 3 shows evidence of LOH in the tumour DNA from patients G11 and D11. However, there was retention of the $165 \mathrm{bp}$ allele in patient D11, who carried the del1100C variant.

\section{DISCUSSION}

In our study, three of the 68 breast cancer patients (4\%; 95\% CI: $1-12 \%)$, carried the $1100 \mathrm{delC}$ variant which we have previously reported to act as a low-penetrance breast cancer susceptibility allele (Meijers-Heijboer et al, 2002). One of these individuals also carried the missense variant, Arg180His. Two other missense variants were also detected in the patients analysed, Arg117Gly and Arg137Gln. Neither of these was detected in a large series of controls implying that they may have a role in the aetiology of breast cancer. On the assumption that they have pathogenic potential, variations in CHEK2 may account for around 7\% (95\%; CI: $2-16 \%$ ) of familial breast cancer cases.

Two previously reported studies have analysed the whole of CHEK2 in familial breast cancer - Allinen et al (2001) studied 79 hereditary breast cancer cases and Sullivan et al (2002) exam- 
ined 45 familial breast cancer cases. Neither found evidence to support a role of sequence variation in CHEK2 in familial breast cancer. However, both studies failed to show that del1100C confers an increased risk of breast cancer.

One of the missense sequence changes we identified involved Arg117 of CHEK2. Arg117 resides within the FHA domain of CHEK2 and is conserved through evolution (Matsuoka et al, 1998). Tumour DNA from the individual with this variant was available to investigate whether loss of heterozygosity is a mechanism by which this variant might confer susceptibility. Allelic imbalance was detected, but the wild type allele was retained and the mutant allele lost. It is possible that the wild type allele has acquired a more lethal somatic mutation and therefore the mutant allele has been preferentially lost or that this variant causes cancer by a mechanism other than by loss of the wild type allele.

Ahn et al (2002) have recently shown that some mutations in the FHA domain negatively affect activation of CHEK2. In response to ionising radiation, ATM phosphorylates Thr-68 of CHEK2 (Ahn et al, 2000). CHEK2 is then autophosphorylated on Thr-383 and Thr-387 in a phospho-threonine 68 ( $\operatorname{Thr}(\mathrm{P})$-68)-dependent manner (Lee and Chung, 2001). Ahn et al (2002) have shown that phosphorylation of Thr- 68 promotes oligomerisation of CHEK2 by serving as a specific ligand for the FHA domain of another CHEK2 molecule. Only catalytically inactive CHEK2 forms oligomers. Ahn et al (2002) have postulated that activation of CHEK2 occurs through oligomerisation of CHEK2 via binding of the Thr- 68 phosphorylated region of one CHEK2 to the FHA domain of another. Oligomerisation of CHEK2 therefore increases the efficiency of transautophosphorylation resulting in the release of active CHEK2 monomers that proceed to enforce checkpoint control. These authors have also shown that Arg117Ala mutation negatively affects autophosphorylation by significantly reducing the ability of CHEK2 to bind to thr (P)-68 molecule. Since the mutation we identified, Arg117Gly, is also a non-conservative substitution it is highly likely to behave in the same way.

\section{REFERENCES}

Ahn J, Schwarz JK, Piwinca-Worms H, Canman CE (2000) Threonine 68 phosphorylation by ataxia telangiectasia mutated is required for efficient activation of Chk2 in response to ionizing radiation. Cancer Res 60: $5934-5936$

Ahn J, Li X, Davis HL, Canman CE (2002) Phosphorylation of Threonine 68 promotes oligomerization and autophosphorylation of the Chk2 protein kinase via the Forkhead-Associated (FHA) domain. J Biol Chem 277: $19389-19395$

Allinen M, Huusko P, Mantyniemi S, Launonen V, Winqvist R (2001) Mutation analysis of the $\mathrm{CHK} 2$ gene in families with hereditary breast cancer. $\mathrm{Br}$ J Cancer 85: 209-212

Antoniou AC, Pharoah PDP, McMullan G, Day NE, Ponder BAJ, Easton DF (2001) Evidence for further breast cancer susceptibility genes in addition to BRCA1 and BRCA2 in a population based study. Genet Epidemiol 21: $1-$ 18

Chaturvedi P, Eng WK, Zhu Y, Mattern MR, Mishra R, Hurle MR, Zhang X, Annan RS, Lu Q, Faucette LF, Scott GF, Li X, Carr SA, Johnson RK, Winkler JD, Zhou BB (1999) Mammalian Chk2 is a downstream effector of the ATM-dependent DNA damage checkpoint pathway. Oncogene 18: 4047 4054

Chehab NH, Malikzay A, Appel M, Halazonetis TD (2000) Chk2/hCds1 functions as a DNA damage checkpoint in $\mathrm{G}(1)$ by stabilizing p53. Genes Dev 14: $289-300$

Easton DF (1999) How many more breast cancer predisposition genes are there. Breast Cancer Res 1: $14-17$

Ganguly A, Rock MJ, Prockcop DJ (1993) Conformation-sensitive gel electrophoresis for rapid detection of single-base differences in double-stranded PCR products and PCR fragments: evidence for solvent-induced bends in DNA heteroduplexes. Proc Natl Acad USA 90: 10325-10329
Tumour DNA from two patients with the 1100delC variant was also available to assess allelic imbalance. In one of the 1100delC cases no LOH was observed. In the other case, D11, as in the case with the Arg117Gly variant, sequencing of exon 10 showed that the wild type allele was retained. However, in this individual there was retention of the microsatellite $165 \mathrm{bp}$ allele that is associated with $1100 \mathrm{delC}$. It is possible that a recombination event may have taken place between the 1100del variant and the D22S275allele.

The CHEK2*1100delC mutation results in truncation of the protein at codon 381 . Hence the functional segment of the protein encompassing amino acids T383 and T387 which are responsible for autophosphorylation will be lost. It is conceivable that if oligomerisation takes place between one normal peptide and one truncated peptide, transautophosphorylation may be not be possible and the normal CHEK2 molecules may still remain bound to the truncated molecules reducing the concentration of the active monomers to respond to DNA damage. Our present investigation suggests that a few rare variants in CHEK2 may confer an increased susceptibility to breast cancer. On the assumption that all the variants we have identified have pathogenic potential then variation in CHEK2 might account for 7\% (95\% CI indicate up to $16 \%$ ) of familial breast cancer. The way in which mutations in CHEK2 cause breast cancer is likely to be through mechanisms other than the loss of heterozygosity that is observed with other classical tumour suppresser genes.

\section{ACKNOWLEDGEMENTS}

We thank the patients that took part in this study and their clinicians. We thank Carolanne Brown and Charlotte Wilson for assistance with data collection. We thank Lynn O'Brien for assistance with putting together the pedigrees. We are grateful for support from the Royal Marsden Hospital NHS Charitable Trust Funds and the Tony Maxse/Hugh Knowles Fund in memory of Georgina Knowles.

Hogervorst F, Cornelis R, Bout M, Van Vliet M, Oosterwijk J, Olmer R, Bakker B, Klijn J, Vasen H, Miejers-Heijboer H, Menko F, Cornelisse C, Den Dunnen J, Devilee P, Van Ommen G-J (1995) Rapid detection of BRCA1 mutations by the Protein Truncation Test. Nat Genet 10: 208-212

Lee JS, Collins KM, Brown AI, Lee CH, Chung JH (2000) hCds1-mediated phosphorylation of BRCA1 regulates the DNA damage response. Nature 404: $201-204$

Lee CH, Chung JH (2001) The hCds1 (CHK2)-FHA domain is essential for a chain of phosphorylation events on hCds1 that is induced by ionizing radiation. J Biol Chem 4: 30537-30541

Matsuoka S, Huang M, Elledge SJ (1998) Linkage of ATM to cell cycle regulation by the Chk2 protein kinase. Science 282: 1893-1897

Meijers-Heijboer H, Ouweland A, Klijn J, Wasielewski M, de Snoo A, Oldenburg R, Hollestelle A, Houben M, Crepin E, Veghel-Plandsoen M, Elstrodt F, Duijn C, Bartels C, Meijers C, Schutte M, McGuffog L, Thompson D, Easton DF, Sodha N, Seal S, Barfoot R, Mangion J, Chang-Claude J, Eccles D, Eeles R, Evans DG, Houlston R, Murday V, Narod S, Peretz T, Peto J, Phelan C, Zhang H, Szabo C, Devilee P, Goldgar D, Futreal PA, Nathanson KL, Weber BL, Rahman N, Stratton MR (2002) Low penetrance familial breast cancer susceptibility due to CHK2 1100delC in non-carriers of BRCA1 or BRCA2 mutations. Nature Genetics 31: $55-59$

Sodha N, Houlston RS, Willaims R, Yuille MA, Mangion J, Eeles RA (2002) A robust method for detecting $\mathrm{CHK} 2 / \mathrm{RAD} 53$ mutations in genomic DNA. Hum Mutat 19: $173-177$

Sullivan A, Yuille MA, Repellin C, Reddy A, Reelfs O, Bell A, Dunne B, Gusterson BA, Osin P, Farrell PJ, Yulug I, Evans A, Ozcelik T, Gasco M, Crook T (2002) Concomitant inactivation of p53 and Chk2 in breast cancer. Oncogene 21: $1316-1324$ 